\title{
Spinodal stratification in micellar films between oil and silica
}

\author{
Gaëlle Rondepierre \\ Laboratoire Sciences et Ingénierie de la Matière Molle, ESPCI Paris, \\ PSL University, Sorbonne Université, CNRS UMR 7615, F-75005 Paris, France \\ Laboratoire Physico-Chimie des Interfaces Complexes, \\ ESPCI Paris, 10 rue Vauquelin, F-75231 Paris, France and \\ TOTAL SA, Pôle d'Etudes et Recherche de Lacq, BP 47, 64170 Lacq, France \\ François Lequeux and Emilie Verneuil \\ Laboratoire Sciences et Ingénierie de la Matière Molle, ESPCI Paris, \\ PSL University, Sorbonne Université, CNRS UMR 7615, F-75005 Paris, France and \\ Laboratoire Physico-Chimie des Interfaces Complexes, \\ ESPCI Paris, 10 rue Vauquelin, F-75231 Paris, France \\ Nicolas Passade-Boupat \\ Laboratoire Physico-Chimie des Interfaces Complexes, \\ Bâtiment CHEMSTARTUP, Route Départementale 817, 64170 Lacq, France and \\ TOTAL SA, Pôle d'Etudes et Recherche de Lacq, BP 47, 64170 Lacq, France \\ Laurence Talini* \\ CNRS, Surface du Verre et Interfaces, Saint-Gobain, 93300 Aubervilliers, France
}

(Dated: May 7, 2021)

\begin{abstract}
We report on the thinning mechanisms of supported films of surfactant (nTAB) solutions above the critical micellar concentration. The films are formed by pressing an oil drop immersed in an aqueous surfactant solution on a silica surface. Depending on the length of the carbon chain of the surfactant and its concentration, two modes of destabilisation of the stratified films are observed. The first one proceeds by heterogeneous nucleation, characterised by the lateral expansion of the domain of lower thickness, as evidenced long ago in suspended micellar films. In addition, the simultaneous stepwise thinning of several domains, called spinodal stratification, is observed here for the first time in supported films. We measure the time evolution of the thickness of the films and we discuss the selection mechanism of each destabilisation mode.
\end{abstract}

\section{INTRODUCTION}

Many processes involve the formation of transient liquid films and their duration are determined by the thinning dynamics of the films [1-4]. For instance, in order to improve the efficiency of oil recovery processes in which oil droplets are pushed by a water flow in a porous medium, water films between oil and solid are needed to persist for a long time. Similarly in microfluidic droplet manipulation, oil droplets are required not to wet the surfaces of the channels.Addition of surfactants to the liquid increases the lifetimes of liquid films because the interactions between the interfaces are then modified and the energy barrier for film rupture increases [5-10].

At surfactant concentrations larger than the critical micellar concentration (CMC), specific effects resulting from the presence of micelles have been observed.In suspended films, often called soap films, which have been the objects of extensive studies, a stepwise thinning mechanism has been evidenced decades ago [11-15]. It results from the stratification of micelles within the film,

\footnotetext{
* laurence.talini@espci.fr
}

each step corresponding to the expulsion of one layer of micelles. It is associated with an oscillating interaction energy as a function of film thickness [14, 16-18]. The step height is given by the pseudo-period of this energy and can be larger than the size of the micelles by one order of magnitude $[16,19,20]$. Thinning proceeds by nucleation events: the film thins down until the disjoining pressure (derivative of the energy with respect to the film thickness $h$ ) is balanced by the applied pressure in the film $[14,16]$. At that stage, its thickness remains constant until a nucleation event occurs, leading to the withdrawal of a micellar layer at a given location and further extending to the whole film [13, 17]. A new metastable state is then reached at a smaller thickness value, corresponding to a new step. The process can repeat several times until final rupture of the film, considerably enhancing its lifetime.

Recently, a thinning process of different nature was observed in suspended micellar films [21]. Instead of thinning at only one given place, multiple patches of smaller thicknesses form, with a well-defined spatial wavelength. By analogy with the spinodal decomposition of binary mixtures, the process was named spinodal stratification. Likewise, it was interpreted as the growth of an instability amplifying thickness 


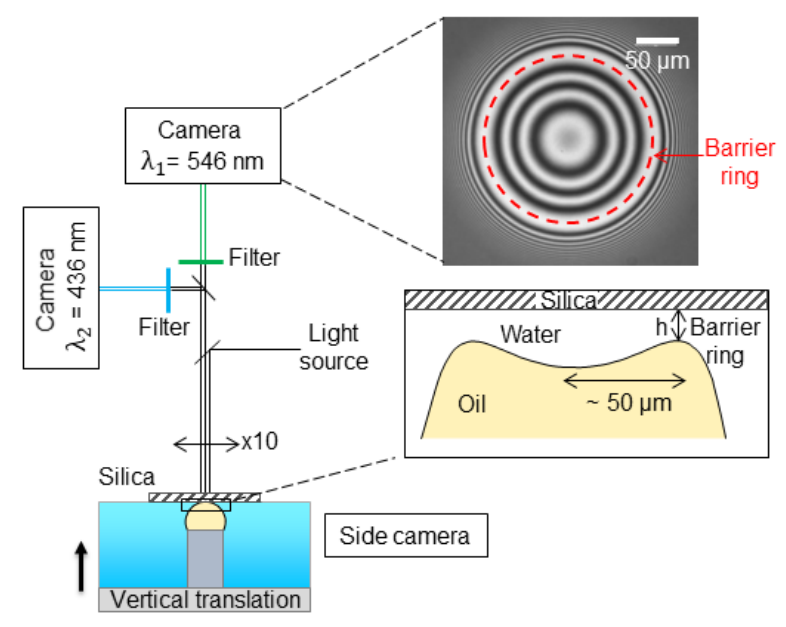

FIG. 1. Schematic representation of the experimental setup, with a close up view of the apex of the drop evidencing the dimpling of the oil/water interface. The interference image (top right) displays equal thickness interference fringes in the dimple and allows accurate thickness measurements. The barrier ring is the location of minimum thickness shown by the dotted red line. All reported thickness measurements have been made at a given point of the barrier ring.

fluctuations. However, the conditions for the occurrence of this spinodal stratification were not clearly identified.

Here, we report the first experimental observations of the stepwise thinning of aqueous supported micellar films not only by heterogeneous nucleation but also by spinodal stratification. Considering the details of the different mechanisms, we clarify the influence of the physical and physico-chemical parameters on the selected mode for thinning, thus providing a full picture for spinodal stratification.

\section{EXPERIMENTAL SYSTEM}

The supported films were obtained by pressing an oil drop, formed in a bath of aqueous surfactant solution, onto a silica surface, as schematized in Fig.1. The pressure applied to the water film is then set by capillary pressure of the oil drop.

The model oil used for the experiments was dodecane (Sigma Aldrich, purity $>99 \%$, density 0.75 ). The solid substrates were uncoated UV-fused plano-convex silica lenses (SPX031) with focal lens $500 \mathrm{~mm}$ from Newport. Due to the presence of silanol groups, the silica surface is negatively charged at the working $\mathrm{pH}$ (between 5 and 7). The cleanliness of the solid surface was a critical parameter for the observation of the water film. Prior to each experiment, the lenses were submitted as received, to a heating cycle at $620^{\circ} \mathrm{C}$ for 15 minutes in a furnace, to burn any organic impurity or surface coating. They were further dusted out under a nitrogen flux. Right before the experiment, each lens was exposed to an ozone atmosphere for 15 minutes and then immersed immediately into the work solution to avoid any contamination.

We chose to study a family of cationic surfactants with varying chain lengths in order to span a large range of micelle concentrations above the CMC. These are the quaternary ammonia commonly called nTAB with $\mathrm{n}$ varying from 12 to 18 . Octadecyltrimethylammonium bromide (OTAB, purity $=98 \%$ ), hexadecyltrimethylammonium bromide (CTAB, purity $>96 \%)$, tetradecyltrimethylammonium bromide (TTAB, purity > 99\%) and dodecyltrimethylammmonium bromide (DTAB, purity $>98 \%$ ) were all purchased from Sigma Aldrich. They were dissolved as received in deionized water with an added background concentration of $10^{-4}$ mol.L $\mathrm{L}^{-1}$ of $\mathrm{NaCl}$ to set the value of the ionic strength in the water phase. Their respective Critical Micellar Concentrations (CMC) were determined from tensiometry experiments (see Table 1) and are in good agreement with the literature [22, 23]. OTAB was studied only slightly above its Krafft temperature $\left(36^{\circ} \mathrm{C}\right)$ [23]. Therefore our results depict only the qualitative behaviour of this surfactant above the CMC.

After approach of the drop, a water film is squeezed between the oil and the silica surface. It deforms the oil/water interface in a shape called dimple and depicted in Figure 1. Water further drains until an equilibrium is reached. This equilibrium is either a metastable situation in which a water film of homogeneous thickness $h_{0}$ persists between the drop and the surface, or the wetting of oil on the surface, corresponding to the global energy minimum. The drop radius $R$ is close to $1 \mathrm{~mm}$, whereas the dimple spreads over $100 \mu \mathrm{m}$, and the water film has a thickness close to $100 \mathrm{~nm}$. In order to image these different scales, we use both a macroscopic side view to observe at the scale of the drop, and a microscope to observe top views of the dimple, as previously described [24].

Optical interferometry is used to measure the thickness of the water film. A high precision dual-wavelength interferometry set-up was developped and is schematically depicted in Fig. 1. Reflection microscopy was used with episcopic illumination provided by a Mercury/Xenon light source and a 10x objective. A beam splitter and a set of bandpass filters $\left(\lambda_{1}=546 \mathrm{~nm}\right.$ and $\left.\lambda_{2}=436 \mathrm{~nm}\right)$ allow acquisition of monochromatic interference images captured by two synchronised cameras. Additional optical elements developed by Hamamatsu allow a fine compensation of chromatism effects in the microscope and synchronized dual-image acquisition.

A transparent PMMA tank was filled with the aque- 
ous solution and placed under the microscope. An oil drop with controlled volume ranging from 0.5 to $1 \mu \mathrm{L}$ was formed at the tip of a polymer tube (PEEK, ID $65 \mu \mathrm{m}$, OD $1.59 \mathrm{~mm}$ ) connected to a syringe. The tank was set to a motorised vertical stage (PI Mikromove), controlling its displacement and velocity, which was set to $15 \mathrm{~mm} . \mathrm{s}^{-1}$. The silica lens was held at the water surface. Finally, side views of the oil droplet were imaged with a camera at $2 \mathrm{fps}$ (resolution of $2.8 \mu \mathrm{m}$ per pixel) and a macro lens, from which the radius of the drop $R$ was measured.

\section{RESULTS}

\section{A. Observations}

Once formed, the drop is approached and further held still close to the solid surface. Due to the difference between the disjoining pressure $\pi(h)$ and the pressure within the $\operatorname{drop} P=2 \gamma / R$, the water film drains. Typically, with $\gamma \approx 5 \mathrm{mN} \cdot \mathrm{m}^{-1}$ and $R \approx 0.5 \mathrm{~mm}, P$ ranges from 15 to $20 \mathrm{~Pa}$. We follow the drainage of nTAB solutions at concentrations above the CMC. The classical images of drainage display equal thickness interference rings centered on the dimple, as can be seen on Fig.1. Without surfactant, water drains out of the dimple, continuously decreasing its thickness, which creates a succession of interference fringes until a uniform coloration spreads over the whole dimple, as previously reported $[24,25]$.

With surfactant solutions of concentrations above the CMC, instead of a continuous water drainage, we observe several sharp thickness variations at the barrier ring, which is the circular region of minimum thickness (see Fig.1). Such thickness variations correspond to stepwise thinning that has been widely studied in suspended films. We present in Fig 2 and 3 the interferometry images for the nTAB surfactants and the evolution of the thickness at a given point of the barrier ring over time.

\section{Classical stratification}

A step-by-step drainage is observed with DTAB solutions at concentrations above 2 CMC. Figure 2 displays an example of the images obtained at $5 \mathrm{CMC}$. A sudden drop of thickness occurs first at only one point of the barrier ring and further extends along it. It is then followed by two other collapses that nucleate and propagate in the same way. The final film of thickness $h_{0}=10 \mathrm{~nm}$ destabilises and wetting eventually occurs about $300 \mathrm{~s}$ after formation of the dimple, as shown in Fig. 2c-d. The thickness $h$ of the film was measured over time at the point marked in red in Fig. 2a and its variations are shown in Fig. 2e. Successive steps are
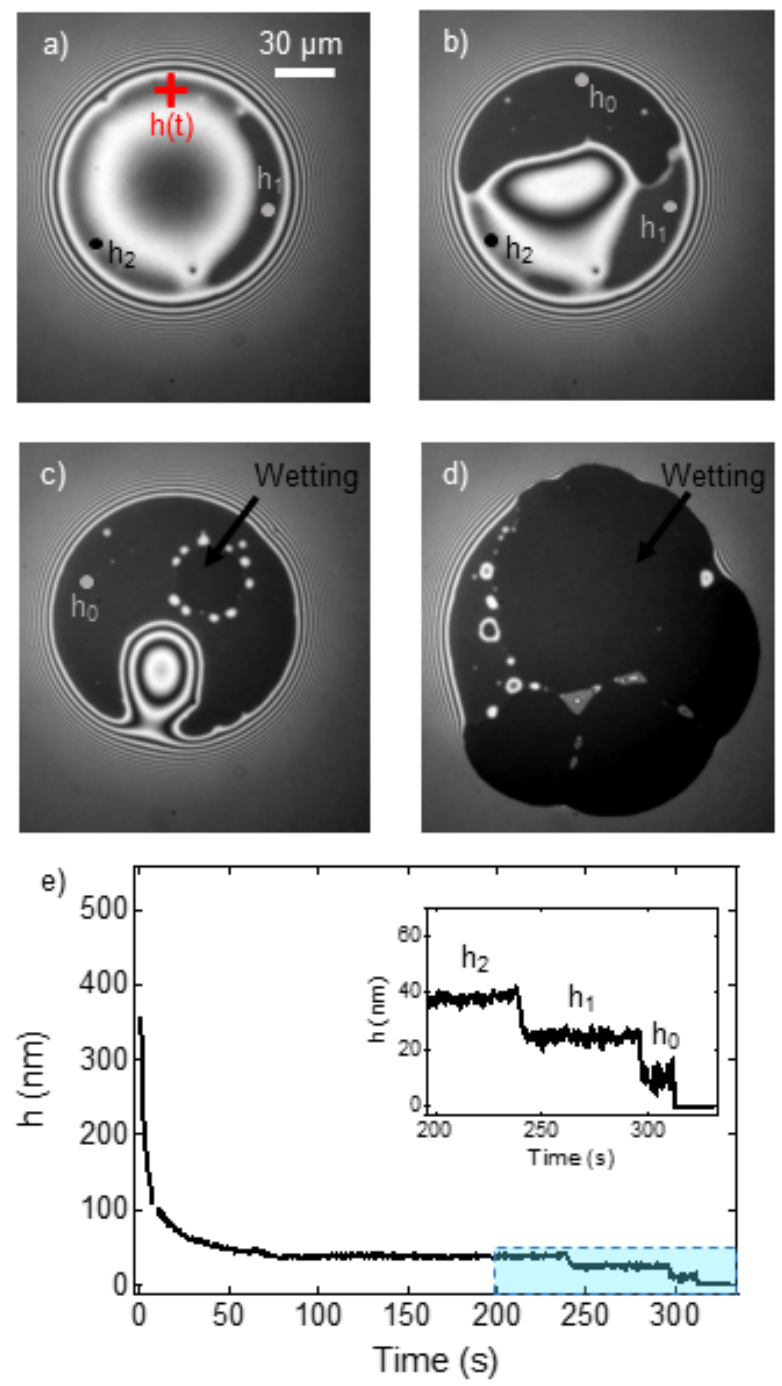

FIG. 2. Interference images of the step-by-step drainage of a water film containing 5 CMC in DTAB a) $\mathrm{t}=197.5 \mathrm{~s}$, b) $\mathrm{t}=302 \mathrm{~s}, \mathrm{c}) \mathrm{t}=308 \mathrm{~s}, \mathrm{~d}) \mathrm{t}=322 \mathrm{~s}$ after the approach, e). The red cross indicates the location in the barrier ring at which thickness $h(t)$ was measured (bottom graph). Steps corresponding to thickness values $h_{2}=38 \mathrm{~nm}, h_{1}=25 \mathrm{~nm}$ and $h_{0}=10 \mathrm{~nm}$ are observed. Inset: close up view of stepwise thinning shown by a rectangle in main graph.

evidenced, with a step height $\Delta h \approx 15 \mathrm{~nm}$.

The same destabilisation mode was also observed for the later thickness steps of films of TTAB solutions at 5 and $10 \mathrm{CMC}$. We show in the following that earlier destabilisation of these TTAB films occurs through a different mechanism.

\section{Spinodal destabilisation}

We now consider the destabilisation of films formed with CTAB solutions. Figure 3a-d displays typical 

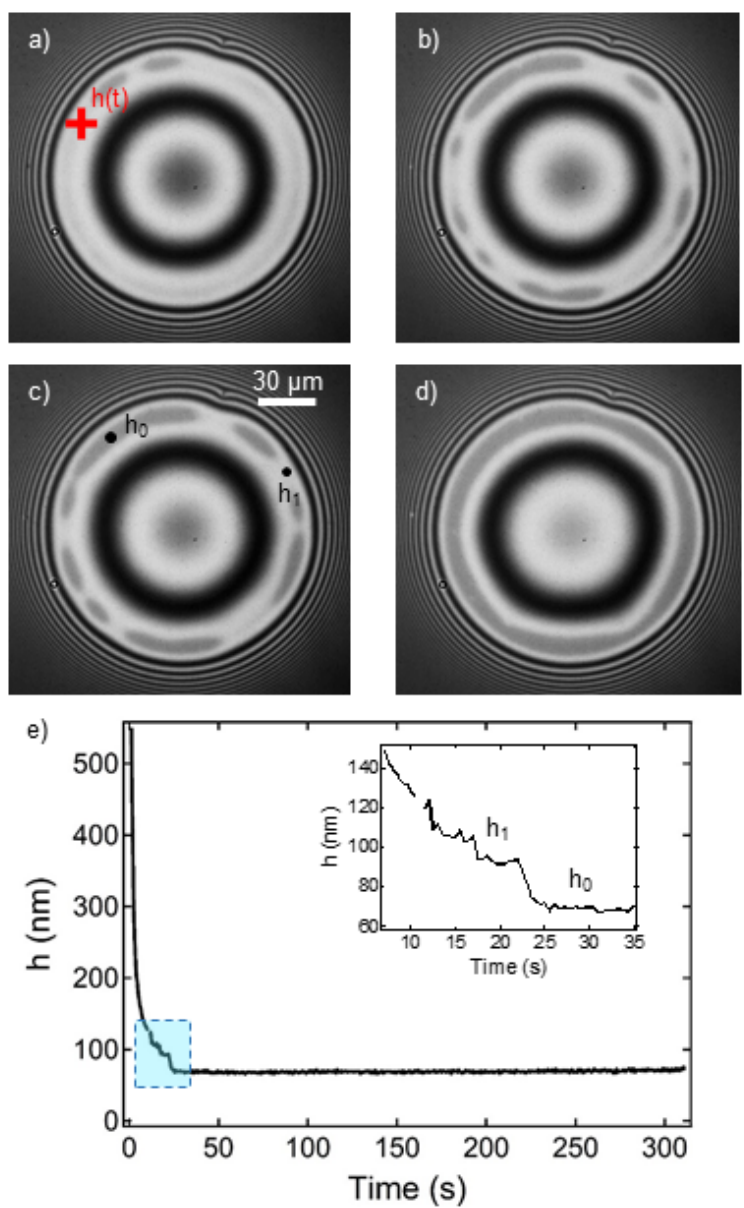

FIG. 3. (Top) Interference images of the step-by-step drainage of a water film containing 2.5 CMC in CTAB a) $\mathrm{t}=22 \mathrm{~s}$, b) $\mathrm{t}=24 \mathrm{~s}, \mathrm{c}) \mathrm{t}=28 \mathrm{~s}, \mathrm{~d}) \mathrm{t}=85 \mathrm{~s}$ after the approach, e). The red cross indicates the location in the barrier ring at which thickness was measured over time (bottom graph). Steps corresponding to thickness values $h_{1}=95 \mathrm{~nm}$ and $h_{0}=$ $70 \mathrm{~nm}$ are observed. Inset: close up view of stepwise thinning shown by a rectangle in main graph.

images of this thinning at a concentration of 2.5 CMC. The corresponding thickness profile is shown in Fig. 3e. When the film thickness reaches $h_{1} \approx 95 \mathrm{~nm}$, a slowing down of drainage is first observed, which is further followed by a steep thickness decrease down to $h_{0}=70 \mathrm{~nm}$. The thickness $h_{0}$ of the barrier ring then remains constant for a few hours and coexists with a central blister (Fig. 3d).

We emphasize that in this case, the triggering of the thickness decrease differs from the heterogeneous nucleation observed with DTAB solutions. As displayed in Fig. 3a-c, several regions of thickness $h_{0}$ appear simultaneously at different places along the barrier ring instead of propagating from one initial position. The regions of thickness $h_{0}$ then grow until the whole barrier ring collapses to $h_{0}$. We will comment later on the interpretation of this destabilisation mechanism but this observation suggests that an instability with a selected wavelength develops, as observed in spinodal destabilisation of liquid films.

A similar pattern as in films of CTAB solutions was observed with OTAB solutions of concentration $5 \mathrm{CMC}$, with only one step of height $\Delta h=40 \mathrm{~nm}$. Interestingly in the case of TTAB solutions, both destabilisation modes could be successively observed: at $2 \mathrm{CMC}$ and, for the first step, at $5 \mathrm{CMC}$ and at $10 \mathrm{CMC}$, similar simultaneous thickness decrease in different regions of the barrier ring were observed as for CTAB. Table 1 summarizes the different characteristics of the stepwise thinning for OTAB, CTAB, TTAB and DTAB at the concentrations studied.

\section{B. Interpretation}

\section{Mechanism of spinodal stratification}

To our knowledge, no report of stepwise thinning of liquid films with surfactants and squeezed between oil and solid can be found in the literature. In past works dealing with similar systems as ours, stepwise thinning is never mentioned [6, 26], possibly because the authors expected a drainage mechanism specific to the dimple geometry. In contrast, stepwise thinning of suspended films has been extensively reported [11-15], with liquids containing either particles or surfactant micelles. In the latter case, for film thicknesses smaller than a hundred nanometers, confinement of highly concentrated solutions results in an organisation of the micelles in structured layers. This phenomenon is referred to as stratification and the disjoining pressure it is associated with is of oscillatory nature.

We expect stratification to occur in confined supported films as well, the difference being that adsorption of surfactants on the solid can take place. This particular feature was characterized by ellipsometry where we measured the thickness of the layer formed by surfactants adsorbed on silica only. A thickness ranging from 1 to $5 \mathrm{~nm}$ was found for the nTABs, which is much smaller than the steps we measured during thinning, and is in agreement with values found in the literature [27]. We conclude that the adsorbed layer does not play any role in the stratification phenomenon. In contrast, it can strongly modify the wetting kinetics of oil on silica [28] but it is not the scope of the present work.

We therefore expect to observe the same phenomena in supported films as in suspended micellar films. As described above, a step-by-step thinning at the barrier ring is observed with some surfactant solutions as classically reported with suspended films. In the following, 


\begin{tabular}{|c|c|c|c|c|c|c|c|}
\hline Surfactant $n \mathrm{TAB}$ & \multicolumn{2}{|c|}{ DTAB } & \multicolumn{3}{|c|}{ TTAB } & CTAB & OTAB \\
\hline$n$ & \multicolumn{2}{|c|}{12} & \multicolumn{3}{|c|}{14} & 16 & 18 \\
\hline $\mathrm{CMC}\left(\mathrm{mol}^{-\mathrm{L}^{-1}}\right)$ & \multicolumn{2}{|c|}{$1.5 \cdot 10^{-2}$} & \multicolumn{3}{|c|}{$3.7 \cdot 10^{-3}$} & $8.8 \cdot 10^{-4}$ & $3 \cdot 10^{-4}$ \\
\hline $\mathrm{C}_{0} / \mathrm{CMC}$ & 2 & 5 & 2 & 5 & 10 & 2.5 & 5 \\
\hline $\mathrm{C}_{0}$-CMC $\left(10^{-3} \mathrm{~mol} \cdot \mathrm{L}^{-1}\right)$ & 15 & 60 & 3.7 & 14.8 & 33.3 & 1.4 & 1.2 \\
\hline Destabilisation mode & $\mathrm{HN}$ & $\mathrm{HN}$ & SS & $\mathrm{SS} / \mathrm{HN}$ & $\mathrm{SS} / \mathrm{HN}$ & SS & SS \\
\hline$d_{H}(\mathrm{~nm})$ & \multicolumn{2}{|c|}{$4.4[28]$} & \multicolumn{3}{|c|}{$5.4[27]$} & $5.7[17]$ & - \\
\hline$N_{a g g}$ & \multicolumn{2}{|c|}{$48[22]-70[26]$} & \multicolumn{3}{|c|}{$55[22]-100[26]$} & $62[22]-95[17]$ & - \\
\hline$\Delta h_{\exp }(\mathrm{nm})$ & 20 & 15 & 20 & 20 & 15 & 25 & 40 \\
\hline$\Delta h_{\text {elec }}(\mathrm{nm})$ & \multicolumn{2}{|c|}{9} & \multicolumn{3}{|c|}{15} & 26 & - \\
\hline$\Delta h_{\text {osm }}(\mathrm{nm})$ & $|18-20|$ & 12 & $29-36$ & $18-22$ & $14-17$ & $41-48$ & - \\
\hline
\end{tabular}

TABLE I. Characteristics of the nTAB surfactants and nature of the observed stepwise thinning at the barrier ring at the different concentrations $C_{0}$ investigated. HN: Heterogeneous Nucleation, SS: Spinodal. $d_{H}$ and $N_{a g g}$ are respectively the micelle hydrodynamical diameters and the aggregation number. The experimental values $\Delta h$ (uncertainty $\pm 2 \mathrm{~nm}$ ) are compared to the theoretical ones obtained when considering the micellar layer thicknesses are set by (i) electrostatic repulsion ( $\Delta h$ elec) (ii) osmotic pressure is the same within the film and in bulk ( $\Delta h$ osm) using eq. (2).

we call that process heterogeneous nucleation.

Recently, Yilixiati et al.[21] evidenced what they called spinodal stratification in freestanding surfactant (SDS) films. They observed the formation of thick-thin spinodal patterns with a characteristic wavelength, similar to the patterns associated with spinodal dewetting. They found spinodal stratification occurred instead of heterogeneous nucleation according to the evaporation conditions in their experiment but they did not identify the reason why a mechanism was selected rather than the other. We attribute the behaviours observed with supported films of TTAB (for the first steps or at smaller concentration), CTAB and OTAB solutions to the same mechanism of spinodal stratification. We suggest its selection is set by the relative values of the imposed pressure and the maxima of the oscillating disjoining pressure.

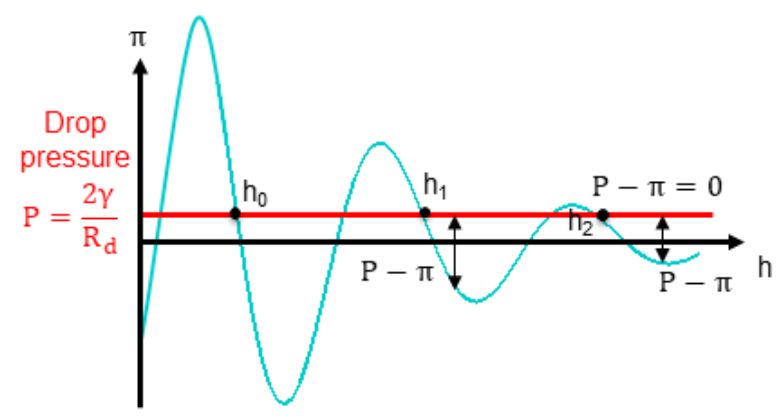

FIG. 4. Theoretical evolution of the oscillatory disjoining pressure $\pi(h)$ with the thickness of the water film $h$ (blue). The red line is the imposed pressure in the drop $2 \gamma / R$ and the driving force of the drainage is the difference $P-\pi(h)$. The values of $h$ at which $P-\pi(h)=0$ are denoted $h_{2}, h_{1}$ and $h_{0}$ and correspond to heterogeneous nucleation events.
We now focus on the variations of disjoining pressure with film thickness in order to understand the difference between heterogeneous nucleation and spinodal stratification. Figure 4 shows typical variations with film thickness of the disjoining pressure resulting from micelles stratification within the film $[14,16,18]$. Initially, the film is thick and drains due to the difference between the applied pressure, corresponding to the capillary pressure in the $\operatorname{drop} P$, and disjoining pressure $\pi(h)$. The driving pressure difference $P-\pi(h)$ is shown in Fig. 4. As drainage proceeds, $h$ decreases and $P-\pi(h)$ varies: it first decreases until it vanishes at a thickness denoted $h_{2}$. At that stage, a metastable state of the film is reached and drainage stops as the applied pressure is balanced by disjoining pressure. However, if a nucleation event allows a local decrease of thickness, the driving pressure difference $P-\pi(h)$ operates again and the drainage resumes, until the film thins down to the thickness at which driving pressure is again equal to zero, denoted $h_{1}$ in Fig. 4. The nucleation event can be either a vibration or some impurities or inhomogeneity on the surface. In this framework, the step height $\Delta h$ we measure corresponds to $\Delta h=h_{2}-h_{1}$ or more generally $h_{i+1}-h_{i}$.

The second destabilization mode, called spinodal stratification can also be explained by considering the disjoining pressure curve. Drainage is still driven by the difference between $P$ and $\pi(h)$. However, as represented in Fig. 5, at a maximum of disjoining pressure, the driving force may become extremely small but remain non-zero. A strong slowing down of drainage is then expected for a film thickness denoted $h_{1}$. As drainage proceeds extremely slowly, the system can reach the dark blue part of the disjoining pressure curve, corresponding to an unstable situation. As theoretically described by Derjaguin, an instability can then develop and grow, inducing thinning of the film at different regions at the 
barrier ring.

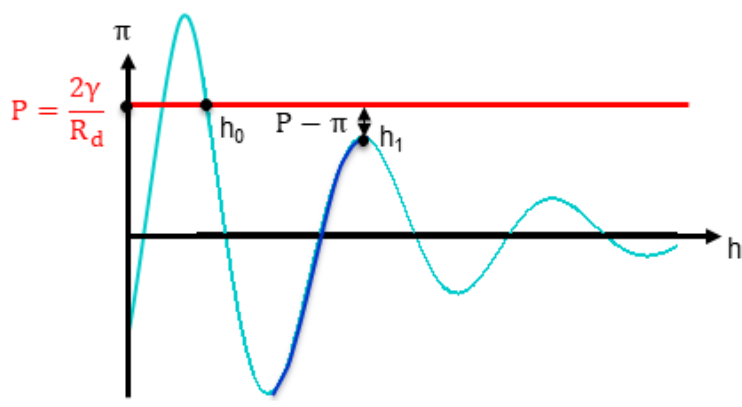

FIG. 5. Theoretical evolution of the oscillatory disjoining pressure $\pi(h)$ with thickness of the surfactant film $h$ (blue). The dark blue zone corresponds to a positive derivative $d \pi / d h$ leading to the development of the instability, characteristic of the spinodal thinning. The red line is the imposed pressure in the drop $2 \gamma / R$ and the driving force of drainage is the difference $P-\pi(h)$.

As most spinodal phenomena, this destabilisation occurs with a characteristic wavelength $\lambda$, which can be related to the interfacial tension between oil and water $\gamma$ and to the slope of $\pi(h)$ in the unstable region. Indeed, the balance between the stabilizing effect of interfacial tension $\gamma$ and the disjoining pressure term $\frac{\partial \pi}{\partial h}$ leads to the selection of a spatial mode characterized by a wavelength $\lambda$ given by Eq. 1 [18] :

$$
\lambda=2 \pi \sqrt{\frac{2 \gamma}{\frac{\partial \pi}{\partial h}}}
$$

For CTAB and OTAB, our experimental observations reveal the existence of such a wavelength selection mechanism. As shown in Fig. 6, the intensity profiles measured along the barrier ring for CTAB and OTAB clearly evidence a spatial wavelength $\lambda$, thereby confirming the spinodal nature of the phenomena observed. From measured values of $\lambda$ and $\gamma$, we can estimate the value of the slope $\frac{\partial \pi}{\partial h}$ at the instability. We find $5 \cdot 10^{8} \mathrm{~Pa} . \mathrm{m}^{-1}$ for CTAB at $2.5 \mathrm{CMC}$ and $1 \cdot 10^{8} \mathrm{~Pa} \cdot \mathrm{m}^{-1}$ for OTAB at 5 CMC. Close to a maximum, disjoining pressure approximately varies as $\Pi_{0} \cos (2 \pi h / \Delta h)$ where the amplitude $\Pi_{0}$ is very close to the imposed pressure $P$. Thus, $\frac{\partial \pi}{\partial h} \approx-\left(2 \pi \Pi_{0} / \Delta h\right) \sin (2 \pi h / \Delta h)$. The pre-factor $\left(2 \pi \Pi_{0} / \Delta h\right)$ is about $10^{10} \mathrm{~Pa} \cdot \mathrm{m}^{-1}$, which is two orders of magnitude larger than the measured value of $\frac{\partial \pi}{\partial h}$. As a result, the selected wavelength must correspond to a thickness value for which the sine term is very small, i.e. which is very close to $h_{1}$ for which pressure is maximum.
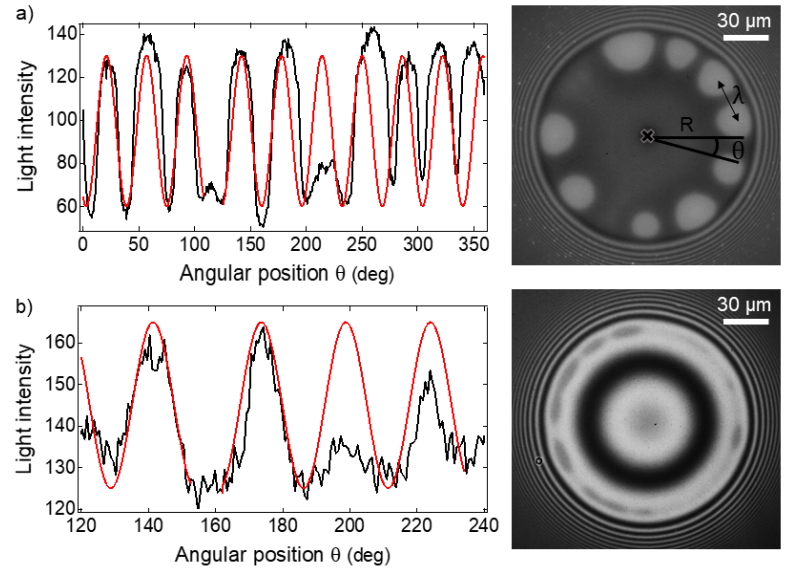

FIG. 6. Black line: Intensity profile at the barrier ring for a) OTAB 5 CMC and b) CTAB 2.5 CMC, evidencing the characteristic wavelength $\lambda$. Red line: sine curve with a spatial period $\lambda$ : a) $\lambda=51 \mu \mathrm{m}$; b) $\lambda=40 \mu \mathrm{m}$.

\section{Selection of the destabilisation mechanism}

In light of the previous results, we now discuss the conditions for each destabilisation mechanism. We expect spinodal stratification to occur for smaller amplitudes of the disjoining pressure. The parameters we vary in the experiment, i.e. length of the carbon chain of the surfactants and their concentration, both result in variations of micelle concentration, which value is not determined precisely. As a result, it is difficult to fully quantify disjoining pressure but trends can be inferred from the quantities we measure in the experiment, among which the heights of the steps $\Delta h$. Their values are reported in Table 1 for each surfactant and can be compared to the existing data in the literature on suspended films. Danov et al. reported stepwise thinning of CTAB at concentrations varying from 10 to 50 CMC [16]. At $10 \mathrm{CMC}$, they measured $25 \mathrm{~nm}$-thick steps. Regarding the final thickness of the film, they showed that it rather corresponds to a solution of diluted micelles than to stratified layers.

The height of the steps is larger than the hydrodynamic diameter of the micelles $d_{H}$ whose values are taken from the literature $[12,16,29]$ and are reported in Table 1. The values of the step height can be compared with the ones predicted within two different frameworks. The first one considers electrostatic repulsions and relates $\Delta h$ to the effective size of the micelles, taking into account the Debye length $\lambda_{D}$ through $\Delta h=d_{H}+2 \lambda_{D}$ [12]. Making the approximation that $\lambda_{D}$ is constant over the concentration range we span, we obtain for CTAB $\Delta h$ $=26 \mathrm{~nm}$, for TTAB $\Delta h=15 \mathrm{~nm}$, and for DTAB $\Delta h$ $=9 \mathrm{~nm}$. These values are in good agreeement with the ones we measured during film thinning (see Table 1).

Danov et al. suggested the average distance $\Delta h$ be- 
tween micelles may alternatively be set by the osmotic pressure of the micelles [16] in the case it is larger than the other pressure terms in the confined film. By assuming the osmotic pressure in the film is the same as in the bulk, the distance between micelles can be equivalently derived from the aggregation number $N_{\text {agg }}$ and the micelles concentration in the bulk $\left(C_{0}-\mathrm{CMC}\right)$ :

$$
N_{a g g}=\left(C_{0}-\mathrm{CMC}\right) \Delta h^{3}
$$

Using aggregation numbers found in the literature and reported in Table 1, we provide estimates for the theoretical values of $\Delta h$ in this framework. The results are presented in Table 1. For CTAB and TTAB at 2 CMC, we find values much larger than the measured ones. As a result, we suggest that in these more dilute surfactant solutions, the average distance between micelles rather results from their charge-induced repulsion. In contrast, for TTAB at 5 and 10 CMC and DTAB, the calculated $\Delta h$ are consistent with the measured values. However, a good agreement is also found between measured $\Delta h$ and the effective radius taking into account micelle repulsion. It is therefore difficult to fully conclude on the relevant effect at stake at larger concentrations, but in any case we can assume $\Delta h=d_{H}+2 \lambda_{D}$.

Comparison of the evolution of $h$ with time for TTAB at different concentrations and in relation with disjoining pressure isotherms, as in Fig. 7 provides hints to understand which destabilisation mode is selected as a function of surfactant concentration. With TTAB solutions, we evidence an increase of the number of steps, with a decreasing height as concentration increases (see Fig 7a). In the literature, this observation is related to a more pronounced structuration when the number of micelles increases [20]. For further understanding, we use the phenomenological expression for disjoining pressure derived by Yilixiati et al. [21] with the following parameters: pseudo-period $\Delta h=22 \mathrm{~nm}$ and $N_{a g g}=80[22,30]$. This gives the curves in Fig.7b, showing the shape of the disjoining pressure at 2, 5 and $10 \mathrm{CMC}$ as a function of $h$.

As predicted, when concentration increases, more steps appear and $\Delta h$ decreases. Indeed, when the concentration decreases, the disjoining pressure curve has fewer oscillations of smaller amplitude, which favours spinodal stratification. We also notice that for spinodal stratification (gray arrows in Fig.7), $h$ keeps on decreasing with time, consistently with the picture we suggest in which the driving pressure difference keeps a finite value eventhough it is small, and therefore in which water drainage never completely stops. In contrast, nucleation events are clearly preceded by thickness plateaus, indicating the driving pressure is strictly zero in that case and drainage stops.

Finally, we comment on the effect of changing the carbon chain length of the surfactant. Nikolov and
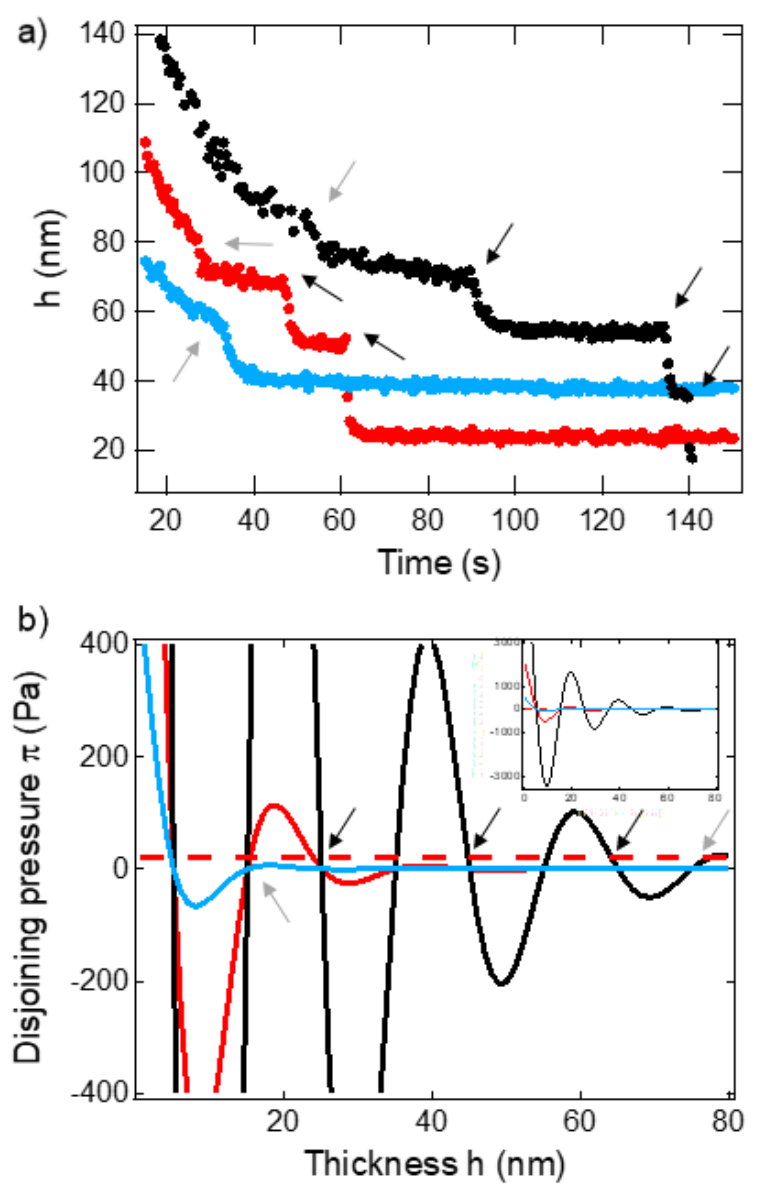

FIG. 7. a) Thickness measured as a function of time at a point of the barrier ring for TTAB at 2 CMC (blue), 5 CMC (red) and $10 \mathrm{CMC}$ (black). The gray arrows indicate occurence of spinodal destabilisation and the black arrows of nucleation event b) Disjoining pressure isotherms for TTAB at 2 CMC (blue), 5 CMC (red) and $10 \mathrm{CMC}$ (black), according to the theoretical expression from [21], with a zoom close to the low pressures. The red dotted line corresponds to the typical experimental drop pressure $P=2 \gamma / R=20 \mathrm{~Pa}$. Arrows indicate the events reported in a). The whole curve is shown in the inset.

Wasan have shown that the effect of the carbon chain length reduces to a concentration effect through the CMC dependence with chain length [29]. Increasing this length results in a decrease of micelle concentration and therefore of the oscillation amplitude of the disjoining pressure [18]. Consequently, spinodal stratification is favored for surfactants with longer chain lengths, consistently with our observations.

In summary, stepwise thinning of the micellar films we study proceeds differently according to the relative values of the imposed pressure and the maximma of the oscillating disjoining pressure. If at a given film thickness, the imposed pressure is smaller than the 
local maximum of disjoining pressure, then thinning is triggered by nucleation events resulting from vibrations, contaminants or defaults of the solid surface. In contrast, if the imposed pressure is slightly larger than a maximum, drainage is almost stopped but an instability can develop driving a spinodal-like mechanism at a given wavelength. Periodic patterns are the signature of this mechanism.

We show our analysis is fully consistent with experimental findings made with nTAB surfactants. Parameters affecting the pseudo-period of the disjoining pressure such as the surfactant chain length and its concentration, can drive a change in the thinning mechanism. Our description does not limit to the homologous series of surfactants we have used but is valid for micellar films of any nature, either free-standing or supported. The fact that heterogeneous nucleation has been more commonly reported than spinodal thinning can probably be ascribed to the low pressure range applied in past studies or possibly to erroneous interpretations [6].

\section{CONCLUSION}

We showed that adding cationic surfactants, at concentrations higher than the CMC, in a water film squeezed between an oil drop and a silica surface strongly enhances the stability of the film, delaying the onset of wetting of oil on the silica surface. When the films is thinner than $100 \mathrm{~nm}$, confined micellar solutions thin out in a stepwise manner. We related this thinning to a stratification phenomenon, often observed in soap films: at high concentration, the confined micelles organize themselves in layers, which induces an oscillatory disjoining pressure with the thickness of the film. Each step corresponds to the expulsion of one layer and can be triggered by either heterogeneous nucleation or spinodal destabilization. Finally, the presence of these layers explains the increased stability of the water film as they induce both steric and electrostatic repulsions between the oil drop and the silica surface. When heterogeneous nucleation is needed to trigger the film thinning, the typical times describing the film drainage diverge and, at low applied pressure, water drainage stops, the film being trapped in a metastable state due to steric repulsions.

\section{ACKNOWLEDGMENTS}

This work benefited from meetings within the French working group GDR CNRS 2019 "Solliciter LA Matière Molle" (SLAMM). This work was part of G. Rondepierre's thesis co-funded by ANRT and Total SA.
[1] D. S. Dimitrov and I. B. Ivanov, Journal of Colloid and Interface Science 64, 97 (1978).

[2] O. Manor, I. U. Vakarelski, X. Tang, S. J. O'Shea, G. W. Stevens, F. Grieser, R. R. Dagastine, and D. Y. C. Chan, Physical Review Letters 101, 024501 (2008).

[3] D. Y. C. Chan, E. Klaseboer, and R. Manica, Soft Matter 7, 2235 (2011).

[4] B. Liu, R. Manica, Q. Liu, E. Klaseboer, Z. Xu, and G. Xie, Physical Review Letters 122, 194501 (2019).

[5] A. K. Chesters and I. B. Bazhlekov, Journal of Colloid and Interface Science 230, 229 (2000).

[6] D. G. Goodall, M. L. Gee, and G. W. Stevens, Langmuir 17, 3784 (2001).

[7] L. Y. Yeo, O. K. Matar, E. Perez de Ortiz, and G. F. Hewitt, Journal of Colloid and Interface Science 257, 93 (2003).

[8] K. D. Danov, in Fluid Mechanics of Surfactant and Polymer Solutions, edited by V. Starov and I. Ivanov (Springer Vienna, Vienna, 2004) pp. 1-38.

[9] B. Dai and L. G. Leal, Physics of Fluids 20, 040802 (2008).

[10] A. Wiertel-Pochopien and J. Zawala, Chemical Engineering \& Technology 42, 1371 (2019).

[11] P. Richetti and P. Kékicheff, Physical Review Letters 68, 1951 (1992).

[12] V. Bergeron and C. J. Radke, Langmuir 8, 3020 (1992).

[13] A. A. Sonin and D. Langevin, Europhysics Letters (EPL) 22, 271 (1993).
[14] P. A. Kralchevsky and N. D. Denkov, Chemical Physics Letters 240, 385 (1995).

[15] K. D. Danov, I. B. Ivanov, K. P. Ananthapadmanabhan, and A. Lips, Advances in Colloid and Interface Science 128-130, 185 (2006).

[16] K. D. Danov, E. S. Basheva, P. A. Kralchevsky, K. P. Ananthapadmanabhan, and A. Lips, Advances in Colloid and Interface Science 168, 50 (2011).

[17] J. Lee, A. Nikolov, and D. Wasan, Journal of Colloid and Interface Science 487, 217 (2017).

[18] S. Yilixiati, R. Rafiq, Y. Zhang, and V. Sharma, ACS Nano 12, 1050 (2018).

[19] V. Bergeron and P. M. Claesson, Advances in Colloid and Interface Science 96, 1 (2002).

[20] R. von Klitzing, E. Thormann, T. Nylander, D. Langevin, and C. Stubenrauch, Advances in Colloid and Interface Science 155, 19 (2010).

[21] S. Yilixiati, E. Wojcik, Y. Zhang, and V. Sharma, Molecular Systems Design \& Engineering 4, 626 (2019).

[22] S. P. Moulik, M. E. Haque, P. K. Jana, and A. R. Das, The Journal of Physical Chemistry 100, 701 (1996).

[23] K. K. Sharker, M. N. Islam, and S. Das, Journal of Surfactants and Detergents 20, 923 (2017).

[24] L. Bluteau, M. Bourrel, N. Passade-Boupat, L. Talini, E. Verneuil, and F. Lequeux, Soft Matter 13, 1384 (2017).

[25] D. G. Goodall, M. L. Gee, and G. W. Stevens, Langmuir 18, 4729 (2002). 
[26] C. S. Tan, M. L. Gee, and G. W. Stevens, Langmuir 19, 7911 (2003), https://doi.org/10.1021/la0270237.

[27] L. Macakova, E. Blomberg, and P. M. Claesson, Langmuir 23, 12436 (2007).

[28] G. Rondepierre, F. De Soete, N. Passade-Boupat, F. Lequeux, L. Talini, L. Limat, and E. Verneuil,
Langmuir 37, 1662 (2021), pMID: 33502209, https://doi.org/10.1021/acs.langmuir.0c02746.

[29] A. D. Nikolov and D. T. Wasan, Journal of Colloid and Interface Science , 1 (1989).

[30] M. J. Rosen and J. T. Kunjappu, in Surfactants and Interfacial Phenomena (John Wiley \& Sons, Ltd, 2012) pp. 123-201. 